University of Nebraska - Lincoln

DigitalCommons@University of Nebraska - Lincoln

U.S. Department of Veterans Affairs Staff

Publications

U.S. Department of Veterans Affairs

8-8-2019

\title{
Vitamin D Supplementation and Prevention of Type 2 Diabetes
}

Anastassios G. Pittas

Bess Dawson-Hughes

Patricia Sheehan

James H. Ware

William C. Knowler

See next page for additional authors

Follow this and additional works at: https://digitalcommons.unl.edu/veterans

This Article is brought to you for free and open access by the U.S. Department of Veterans Affairs at DigitalCommons@University of Nebraska - Lincoln. It has been accepted for inclusion in U.S. Department of Veterans Affairs Staff Publications by an authorized administrator of DigitalCommons@University of Nebraska - Lincoln. 


\section{Authors}

Anastassios G. Pittas, Bess Dawson-Hughes, Patricia Sheehan, James H. Ware, William C. Knowler, Vanita R. Aroda, Irwin Brodsky, Lisa Ceglia, Chhavi Chadha, Ranee Chatterjee, Cyrus Desouza, Rowena Dolor, John Foreyt, Paul Fuss, Adline Ghazi, Daniel S. Hsia, Karen C. Johnson, Sangeeta R. Kashyap, Sun Kim, Erin S. LeBlanc, Michael R. Lewis, Emilia Liao, Anne Peters, Lawrence S. Phillips, Richard Pratley, Philip Raskin, Neda Rasouli, David Robbins, Clifford Rosen, Ellen M. Vickery, Myrlene Staten, and Jason Nelson 


\title{
Vitamin D Supplementation and Prevention of Type 2 Diabetes
}

\author{
Anastassios G. Pittas, M.D., Bess Dawson-Hughes, M.D., \\ Patricia Sheehan, R.N., M.P.H., M.S., James H. Ware, Ph.D.,** \\ William C. Knowler, M.D., Dr.P.H., Vanita R. Aroda, M.D., Irwin Brodsky, M.D., \\ Lisa Ceglia, M.D., Chhavi Chadha, M.D., Ranee Chatterjee, M.D., M.P.H., \\ Cyrus Desouza, M.B., B.S., Rowena Dolor, M.D., John Foreyt, Ph.D., \\ Paul Fuss, B.A., Adline Ghazi, M.D., Daniel S. Hsia, M.D., \\ Karen C. Johnson, M.D., M.P.H., Sangeeta R. Kashyap, M.D., Sun Kim, M.D., \\ Erin S. LeBlanc, M.D., M.P.H., Michael R. Lewis, M.D., Emilia Liao, M.D., \\ Lisa M. Neff, M.D., Jason Nelson, M.P.H., Patrick O'Neil, Ph.D., Jean Park, M.D., \\ Anne Peters, M.D., Lawrence S. Phillips, M.D., Richard Pratley, M.D., \\ Philip Raskin, M.D., Neda Rasouli, M.D., David Robbins, M.D., \\ Clifford Rosen, M.D., Ellen M. Vickery, M.S., and Myrlene Staten, M.D., \\ for the D2d Research Group广
}

The authors' affiliations are listed in the Appendix. Address reprint requests to Dr. Pittas at the Division of Endocrinology, Diabetes, and Metabolism, Tufts Medical Center, 800 Washington St., Box 268, Boston, MA 02111, or at apittas@ tuftsmedicalcenter.org.

*Deceased.

$\dagger$ A list of the members of the D2d Research Group is provided in the Supplementary Appendix, available at NEJM.org.

This article was published on June 7, 2019, and updated on July 18, 2019, at NEJM.org.

N Engl J Med 2019;381:520-30. DOI: 10.1056/NEJMoa1900906

Copyright (c) 2019 Massachusetts Medical Society.

This document is a U.S. government work and is not subject to copyright in the United States.

\section{A BSTRACT}

\section{BACKGROUND}

Observational studies support an association between a low blood 25-hydroxyvitamin D level and the risk of type 2 diabetes. However, whether vitamin D supplementation lowers the risk of diabetes is unknown.

\section{METHODS}

We randomly assigned adults who met at least two of three glycemic criteria for prediabetes (fasting plasma glucose level, 100 to $125 \mathrm{mg}$ per deciliter; plasma glucose level 2 hours after a 75-g oral glucose load, 140 to $199 \mathrm{mg}$ per deciliter; and glycated hemoglobin level, 5.7 to $6.4 \%$ ) and no diagnostic criteria for diabetes to receive $4000 \mathrm{IU}$ per day of vitamin $\mathrm{D}_{3}$ or placebo, regardless of the baseline serum 25-hydroxyvitamin D level. The primary outcome in this time-to-event analysis was new-onset diabetes, and the trial design was event-driven, with a target number of diabetes events of 508 .

\section{RESULTS}

A total of 2423 participants underwent randomization (1211 to the vitamin D group and 1212 to the placebo group). By month 24, the mean serum 25-hydroxyvitamin D level in the vitamin D group was 54.3 ng per milliliter (from 27.7 ng per milliliter at baseline), as compared with $28.8 \mathrm{ng}$ per milliliter in the placebo group (from $28.2 \mathrm{ng}$ per milliliter at baseline). After a median follow-up of 2.5 years, the primary outcome of diabetes occurred in 293 participants in the vitamin D group and 323 in the placebo group (9.39 and 10.66 events per 100 person-years, respectively). The hazard ratio for vitamin $\mathrm{D}$ as compared with placebo was $0.88(95 \%$ confidence interval, 0.75 to $1.04 ; \mathrm{P}=0.12)$. The incidence of adverse events did not differ significantly between the two groups.

\section{CONCLUSIONS}

Among persons at high risk for type 2 diabetes not selected for vitamin $\mathrm{D}$ insufficiency, vitamin $\mathrm{D}_{3}$ supplementation at a dose of $4000 \mathrm{IU}$ per day did not result in a significantly lower risk of diabetes than placebo. (Funded by the National Institute of Diabetes and Digestive and Kidney Diseases and others; D2d ClinicalTrials.gov number, NCT01942694.) 
M

ORE THAN 84 MILLION ADULTS IN the United States have an increased risk of type 2 diabetes, based on a fasting glucose or glycated hemoglobin level above the normal range but below the threshold for diabetes. ${ }^{1}$ Persons at high risk for type 2 diabetes who are overweight or obese and who have elevated fasting glucose levels and glucose intolerance (according to a 75-g oral glucose-tolerance test) can slow progression to diabetes with lifestyle changes. ${ }^{2}$ However, achieving and maintaining sufficient lifestyle change is challenging, and the residual risk of diabetes remains elevated, even after successful weight loss.

Over the past decade, a low blood 25-hydroxyvitamin $\mathrm{D}$ level has emerged as a possible risk factor for type 2 diabetes, and vitamin $\mathrm{D}$ supplementation has been proposed as a potential intervention to lower diabetes risk. ${ }^{3,4}$ The hypothesis that vitamin D status may influence the risk of type 2 diabetes is biologically plausible, because both impaired pancreatic beta-cell function and insulin resistance have been reported with low blood 25-hydroxyvitamin D levels. ${ }^{5}$ Observational studies support an association between a low blood 25-hydroxyvitamin D level and the risk of diabetes. ${ }^{6}$ In short-term mechanistic studies, vitamin D supplementation improved the disposition index, a measure of pancreatic beta-cell function, by $40 \%{ }^{7}$ However, whether vitamin $\mathrm{D}$ supplementation lowers the risk of diabetes is unclear. ${ }^{8-10}$ The Vitamin D and Type 2 Diabetes (D2d) trial was conducted to test whether vitamin D supplementation reduces the risk of type 2 diabetes among adults at high risk for the disorder.

\section{METHODS}

\section{TRIAL DESIGN}

This randomized, double-blind, placebo-controlled clinical trial evaluated the safety and efficacy of oral administration of vitamin $\mathrm{D}_{3}$ (cholecalciferol; 4000 IU per day) for diabetes prevention in adults at high risk for type 2 diabetes. ${ }^{11}$ The trial protocol (available with the full text of this article at NEJM.org) was designed by the planning committee and the primary sponsor ( $\mathrm{Na}-$ tional Institute of Diabetes and Digestive and Kidney Diseases) without input from manufacturers $^{11}$ and involved collaboration among 22 academic medical centers in the United States (https://d2dstudy.org/sites). A sponsor-appointed data and safety monitoring board approved the protocol and provided independent monitoring of the trial. The institutional review board at each clinical site also approved the protocol, and all the participants provided written informed consent. The data were collected by trial-site personnel and stored in an electronic data-capture database. The statistical team at the coordinating center analyzed the data and vouches for its accuracy. All the authors vouch for the accuracy and completeness of the data and for the fidelity of the trial to the protocol. They also contributed to the interpretation of the results and the preparation, review, and approval of the manuscript and made the decision to submit the manuscript for publication.

No pharmaceutical manufacturers contributed to the planning, design, or conduct of the trial. Trial pills were purchased from an independent nutritional-supplement manufacturing company that has no association with any members of the D2d Research Group.

\section{PARTICIPANTS}

Participants met at least two of three glycemic criteria for prediabetes as defined by the 2010 American Diabetes Association (ADA) guidelines: fasting plasma glucose level, 100 to $125 \mathrm{mg}$ per deciliter (5.6 to $6.9 \mathrm{mmol}$ per liter); plasma glucose level 2 hours after a 75-g oral glucose load, 140 to $199 \mathrm{mg}$ per deciliter ( 7.8 to $11.0 \mathrm{mmol}$ per liter); and glycated hemoglobin level, 5.7 to $6.4 \%$ (39 to $47 \mathrm{mmol}$ per mole). ${ }^{12}$ Other inclusion criteria were an age of 30 years or older ( 25 years or older for American Indians, Alaska Natives, or Native Hawaiians or other Pacific Islanders) and a body-mass index (BMI, the weight in kilograms divided by the square of the height in meters) of 24 to 42 (22.5 to 42 for Asian Americans). A low serum 25-hydroxyvitamin D level was not an inclusion criterion.

Key exclusion criteria were any glycemic criterion in the diabetes range, ${ }^{12}$ factors (other than hyperglycemia and race) affecting the glycated hemoglobin level, use of diabetes or weight-loss medications, or use of supplements containing vitamin $\mathrm{D}$ at a dose of more than 1000 IU per day or calcium at a dose of more than $600 \mathrm{mg}$ per day. For a complete list of eligibility criteria, see the Supplementary Appendix (available at NEJM.org). The recruitment process relied primarily on electronic-health-record identification of potentially eligible adults who were then 
screened in person and, if qualified, had a second screening visit to determine final eligibility according to measured fasting plasma glucose, 2-hour post-load plasma glucose, and glycated hemoglobin at the central laboratory of the trial. ${ }^{13}$

\section{INTERVENTION AND PROCEDURES}

Participants were randomly assigned to take a single, once-daily soft-gel pill containing either 4000 IU of vitamin $\mathrm{D}_{3}$ or matching placebo. Randomization was block-stratified according to trial site, BMI $(<30$ or $\geq 30)$, and race (white or nonwhite). Participants received a bottle of trial pills at the time of randomization and every 6 months thereafter. Bottles with unused pills were returned at each visit to estimate adherence.

To maximize the ability of the trial to observe a treatment effect, participants were asked to refrain from using diabetes-specific or weightloss medications during the trial and to limit the use of outside-of-trial vitamin D to 1000 IU per day from all supplements, including multivitamins. Because of concern that high intake of calcium from supplements may be associated with adverse outcomes, participants were asked to limit calcium supplements to $600 \mathrm{mg}$ per day. During the trial, participants were provided with information on diabetes prevention through information sheets and twice-yearly group meetings.

Follow-up visits occurred at month 3 , month 6 , and twice per year thereafter. Midway between the in-person visits, an interim contact (telephone or email) took place. All visits and contacts were designed to promote retention, encourage adherence to the trial regimen, and assess for diabetes, occurrence of adverse events, and use of high-dose vitamin D supplements, diabetes medications, and weight-loss medications.

\section{OUTCOMES}

The primary outcome in this time-to-event analysis was new-onset diabetes, based on annual glycemic testing of fasting plasma glucose, glycated hemoglobin, and 2-hour post-load plasma glucose and semiannual testing of fasting plasma glucose and glycated hemoglobin. If two or three of the glycemic measures met the 2010 ADA thresholds for diabetes, ${ }^{12}$ the participant was considered to have met the diabetes outcome. When only the measure for fasting plasma glucose or glycated hemoglobin met the thresh- old, confirmatory testing was performed for the positive measure within 8 weeks. If only the measure for 2-hour post-load plasma glucose met the threshold, then a 75-g oral glucose-tolerance test to reassess all three glycemic measures was repeated. If the repeat measure was positive or both fasting plasma glucose and glycated hemoglobin were positive (in the case of a repeat oral glucose-tolerance test), then the participant was considered to have met the diabetes outcome. A diagnosis of diabetes that was made outside the trial was validated by in-trial laboratory testing or adjudicated by an independent clinical-outcomes committee.

During the trial, research staff, caregivers, and participants were unaware of glycemic test results until a participant met the diabetes outcome. Safety was assessed by means of participant report and annual fasting measurements of serum calcium, serum creatinine, and morning spot urine calcium:creatinine ratio (a rough estimate of urine calcium excretion). ${ }^{14}$

\section{LABORATORY TESTING}

Serum calcium and creatinine were analyzed locally at each site, and the estimated glomerular filtration rate was calculated. ${ }^{15}$ Other blood and urine specimens were processed locally and shipped to the central laboratory. Glycated hemoglobin was measured with the use of an ion-exchange high-performance liquid chromatography method certified by the National Glycohemoglobin Standardization Program. ${ }^{16}$ Plasma glucose was measured with the use of a hexokinase method. Stored serum samples from the baseline, month 12, and month 24 visits were used to measure 25 -hydroxyvitamin $\mathrm{D}$ by liquid chromatography-tandem mass spectrometry validated by a quarterly proficiency-testing program administered by the Vitamin D External Quality Assessment Scheme. ${ }^{17,18}$

\section{STATISTICAL ANALYSIS}

The trial was designed as an event-driven trial with a target of 508 diabetes events and a total sample size of 2382 participants assigned equally to the vitamin D group and placebo group, on the basis of a hypothesized hazard ratio of 0.75 in the vitamin D group, an incidence of diabetes of $10 \%$ per year in the placebo group, a type I error rate of 0.0501 (with a single interim analy- 
sis taken into account), a power of $90 \%$, a recruitment period of 2 years, a trial duration of 4 years, and a withdrawal rate of $5 \%$ per year of follow-up. ${ }^{19}$

Intention-to-treat analyses compared groups defined by the randomization procedure and included all participants irrespective of adherence to the assigned intervention or to the protocol (e.g., use of diabetes or weight-loss medications). Follow-up time for all analyses was calculated as the time from randomization until the occurrence of the primary outcome, death, withdrawal, or last follow-up encounter free from diabetes. No imputation was performed for missing data, but we conducted a sensitivity analysis to assess for noninformative censoring of incomplete data (see the Supplementary Appendix).

Because the use of a diabetes-specific medication would be considered a "competing event" for the primary outcome, we prespecified a sensitivity analysis in which the primary outcome was the time to new-onset diabetes according to trial criteria or use of a diabetes-specific medication. As planned in the protocol, we conducted an exploratory per-protocol analysis that censored follow-up data when a participant stopped the trial pills, started a diabetes or weight-loss medication, or took out-of-trial vitamin D from supplements above the trial limit of $1000 \mathrm{IU}$ per day.

The protocol specified that this event-driven trial would continue until the required number of diabetes events (508) was reached. A prespecified interim analysis for the data and safety monitoring board to examine harm or superior efficacy with the use of a Haybittle-Peto bound$\operatorname{ary}^{20}$ was conducted when approximately $70 \%$ of the required events (364 of 508) had accrued, and the data and safety monitoring board recommended that the trial proceed to its planned conclusion. Because the efficiency of event-driven trials is increased by stopping when the required number of events is achieved, ${ }^{21}$ we conducted blinded monitoring of event count and specified that when the trial was within approximately 2 months of reaching 508 events, the subsequent scheduled follow-up visit for each participant would be considered the last visit. All events that occurred during the trial, including those that occurred after the target of 508 events was reached, were used to generate the primary results.

Kaplan-Meier estimates were plotted for each group. Cox proportional-hazards models were used to calculate the hazard ratio for new-onset diabetes between the two groups. ${ }^{22}$ The model included group assignment as its main predictor variable and the stratification variables (trial site, BMI, and race). We also show a model without the stratification variables. Comparisons between the two groups at baseline and with respect to the rate of withdrawal, discontinuation of trial pills, use of diabetes or weight-loss medications, and supplemental intake above the trial limit used Fisher's exact test, the chi-square test, the Wilcoxon rank-sum test, or the pooled-variance t-test.

Variability of response to vitamin D supplementation was assessed in prespecified subgroups defined by key baseline variables. Rates of adverse events were compared between the two groups. When evaluating the significance of the prespecified subgroup analyses, we used the Hochberg sequential procedure to adjust for multiple comparisons, if necessary. No adjustments were made for the safety analyses or the planned exploratory or post hoc analyses for the primary outcome; therefore, only point estimates and 95\% confidence intervals are presented without $P$ values.

\section{RESULTS}

\section{PARTICIPANTS}

From October 2013 through February 2017, a total of 7133 persons were screened (Fig. 1), and 2423 were randomly assigned to receive vitamin D (1211 participants) or placebo (1212 participants); these participants were included in the intentionto-treat population (Table 1, and Table S1 in the Supplementary Appendix). A total of $44.8 \%$ of the participants were women, $33.3 \%$ were of nonwhite race, and $9.3 \%$ were of Hispanic ethnic background. ${ }^{24}$ The participants had a mean age of 60.0 years, a mean BMI of 32.1, and a mean glycated hemoglobin level of $5.9 \%$ (48 mmol per mole). A total of $84.2 \%$ of the participants met the glycemic criteria for both fasting plasma glucose and glycated hemoglobin; approximately one third met all three glycemic criteria.

The last trial encounter was in November 2018. In the two groups, the median follow-up was 2.5 years (interquartile range, 1.9 to 3.5 [vitamin D] and 1.7 to 3.5 [placebo]). Before reaching a pri- 


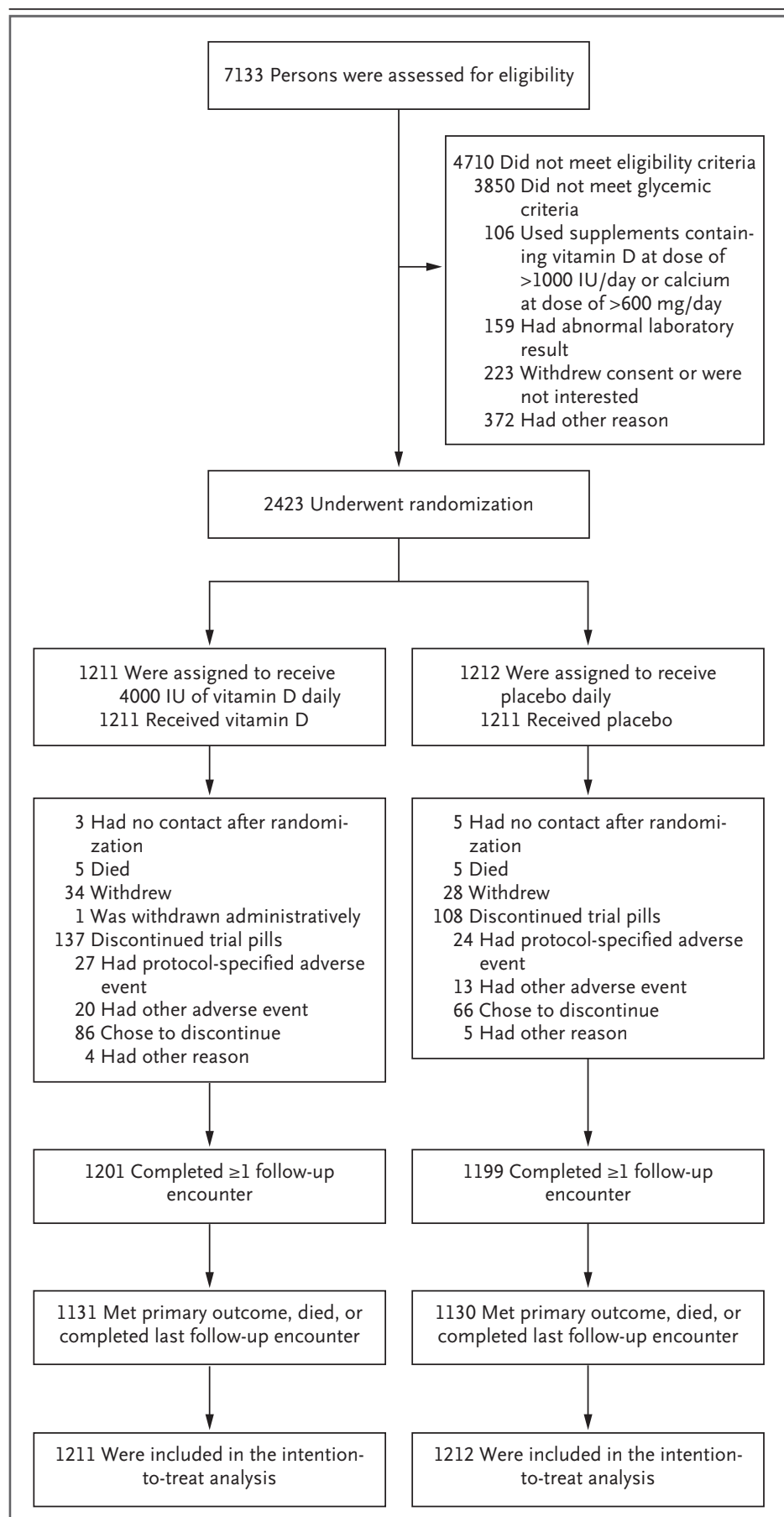

Figure 1. Screening, Randomization, and Follow-up.

One participant in the vitamin $\mathrm{D}$ group was withdrawn administratively after a clinical site closed down early in the trial. Protocol-specified adverse events that led to discontinuation of the trial pills were hypercalcemia, a fasting urine calcium:creatinine ratio of more than 0.375 , a low estimated glomerular filtration rate, and nephrolithiasis. Of the 2423 participants who underwent randomization, 14 ( 9 in the vitamin $D$ group and 5 in the placebo group) were subsequently found not to meet all eligibility criteria. mary outcome event, 10 participants $(5$ in each group) died, and 62 (34 in the vitamin D group and 28 in the placebo group) withdrew consent (Fig. 1). In total, $99.1 \%$ of the cohort (1201 participants in the vitamin D group and 1199 in the placebo group) contributed follow-up data, through either a visit that included central-laboratory testing or a nonvisit encounter to capture a diagnosis of diabetes outside the trial.

The mean baseline level of serum 25-hydroxyvitamin D was $28.0 \mathrm{ng}$ per milliliter $(69.9 \mathrm{nmol}$ per liter), with no significant difference between the two groups; $78.3 \%$ of the participants had a level equal to or greater than $20 \mathrm{ng}$ per milliliter (50 nmol per liter) (Table 1). The mean 25-hydroxyvitamin $\mathrm{D}$ levels in the vitamin $\mathrm{D}$ group at month 12 (52.3 ng per milliliter [130.5 nmol per liter]) and month 24 (54.3 ng per milliliter [135.5 nmol per liter]) were higher than those in the placebo group (28.1 ng per milliliter [70.1 nmol per liter] and $28.8 \mathrm{ng}$ per milliliter [71.9 nmol per liter], respectively) (Fig. S1 in the Supplementary Appendix).

\section{PRIMARY OUTCOME}

By the end of the trial, diabetes had developed in 616 patients. New-onset diabetes (the primary outcome) occurred in 293 participants (273 cases diagnosed by trial-specific laboratory testing and 20 diagnosed by adjudication) in the vitamin $\mathrm{D}$ group and 323 patients (305 cases diagnosed by trial-specific laboratory testing and 18 diagnosed by adjudication) in the placebo group ( 9.39 events and 10.66 events per 100 person-years, respectively). The hazard ratio in the vitamin $\mathrm{D}$ group was 0.88 (95\% confidence interval [CI], 0.75 to 1.04; $\mathrm{P}=0.12$ ) (Fig. 2). When the stratification variables were not included in the model, the hazard ratio in the vitamin $\mathrm{D}$ group was 0.87 ( $95 \%$ CI, 0.75 to 1.02 ). In a sensitivity analysis to account for missing data, the hazard ratio did not change substantially (see the Supplementary Appendix).

In the sensitivity analysis in which diabetes was defined as new-onset diabetes according to trial criteria or the use of a diabetes-specific medication, the hazard ratio in the vitamin $\mathrm{D}$ group was 0.88 (95\% CI, 0.75 to 1.02). The results of the subgroup analyses were consistent with the findings of the main analysis; there was no apparent heterogeneity of treatment effect across the prespecified subgroups (Fig. 3). 


\begin{tabular}{|c|c|c|c|}
\hline Characteristic & $\begin{array}{l}\text { Overall } \\
(\mathrm{N}=2423)\end{array}$ & $\begin{array}{l}\text { Vitamin D } \\
(\mathrm{N}=1211)\end{array}$ & $\begin{array}{l}\text { Placebo } \\
(\mathrm{N}=1212)\end{array}$ \\
\hline \multicolumn{4}{|l|}{ Demographic } \\
\hline Age $-y r$ & $60.0 \pm 9.9$ & $59.6 \pm 9.9$ & $60.4 \pm 10.0$ \\
\hline Female sex — no. (\%) & $1086(44.8)$ & $541(44.7)$ & $545(45.0)$ \\
\hline \multicolumn{4}{|l|}{ Race - no. (\%)† } \\
\hline Asian & $130(5.4)$ & $66(5.5)$ & $64(5.3)$ \\
\hline Black & $616(25.4)$ & $301(24.9)$ & $315(26.0)$ \\
\hline White & $1616(66.7)$ & $810(66.9)$ & $806(66.5)$ \\
\hline Other & $61(2.5)$ & $34(2.8)$ & $27(2.2)$ \\
\hline Hispanic or Latino ethnic group — no. (\%) $\dagger$ & $225(9.3)$ & $120(9.9)$ & $105(8.7)$ \\
\hline Body-mass index & $32.1 \pm 4.5$ & $32.0 \pm 4.5$ & $32.1 \pm 4.4$ \\
\hline \multicolumn{4}{|l|}{ Laboratory assessments } \\
\hline Fasting plasma glucose $-\mathrm{mg} / \mathrm{dl}$ & $107.9 \pm 7.4$ & $108.0 \pm 7.4$ & $107.8 \pm 7.4$ \\
\hline 2- $\mathrm{Hr}$ post-load plasma glucose $-\mathrm{mg} / \mathrm{dl}$ & $137.2 \pm 34.3$ & $136.9 \pm 34.3$ & $137.6 \pm 34.3$ \\
\hline Glycated hemoglobin — \% & $5.9 \pm 0.2$ & $5.9 \pm 0.2$ & $5.9 \pm 0.2$ \\
\hline \multicolumn{4}{|l|}{ Serum 25-hydroxyvitamin D } \\
\hline Mean - ng/ml & $28.0 \pm 10.2$ & $27.7 \pm 10.2$ & $28.2 \pm 10.1$ \\
\hline \multicolumn{4}{|l|}{ Distribution — no./total no. (\%) } \\
\hline$<12 \mathrm{ng} / \mathrm{ml}$ & $103 / 2422(4.3)$ & $60 / 1211(5.0)$ & $43 / 1211(3.6)$ \\
\hline $12-19 \mathrm{ng} / \mathrm{ml}$ & $422 / 2422(17.4)$ & $216 / 1211(17.8)$ & $206 / 1211(17.0)$ \\
\hline $20-29 \mathrm{ng} / \mathrm{ml}$ & $876 / 2422(36.2)$ & $453 / 1211(37.4)$ & $423 / 1211(34.9)$ \\
\hline$\geq 30 \mathrm{ng} / \mathrm{ml}$ & $1021 / 2422(42.2)$ & $482 / 1211(39.8)$ & $539 / 1211(44.5)$ \\
\hline
\end{tabular}

* Plus-minus values are means \pm SD. Percentages may not total 100 because of rounding. To convert the values for glucose to millimoles per liter, multiply by 0.05551 . To convert the values for 25 -hydroxyvitamin $D$ to nanomoles per liter, multiply by 2.496 .

$\dagger$ Race and ethnic group were reported by the participant. The category "other" includes American Indian or Alaska Native; Native Hawaiian or other Pacific Islander; and other race. Ethnic group includes any race.

TCategories of serum 25-hydroxyvitamin D are based on the 2010 Dietary Reference Intakes for calcium and vitamin D recommended by the Food and Nutrition Board of the Institute of Medicine. ${ }^{23}$

In a post hoc analysis of data from participants with a baseline 25-hydroxyvitamin $\mathrm{D}$ level of less than 12 ng per milliliter (30 nmol per liter) (103 participants), the hazard ratio in the vitamin $\mathrm{D}$ group was 0.38 ( $95 \%$ CI, 0.18 to 0.80). Among those with a baseline 25-hydroxyvitamin D level equal to or greater than $12 \mathrm{ng}$ per milliliter (2319 participants), the hazard ratio in the vitamin D group was 0.92 (95\% CI, 0.78 to 1.08).

\section{ADHERENCE}

A total of 170 participants (14.0\%) in the vitamin D group and 172 (14.2\%) in the placebo group stopped trial pills, took diabetes or weightloss medications, or took outside-of-trial vita- min D supplements above the trial limit before the diagnosis of diabetes. During the trial, more participants in the placebo group than in the vitamin D group started diabetes or weight-loss medications (Fig. S2 in the Supplementary Appendix). Although overall adherence to the trial regimen was high $(85.8 \%$ of prescribed pills were taken), more participants in the vitamin $\mathrm{D}$ group (11.3\%) than in the placebo group (8.9\%) stopped trial pills (difference, 2.4 percentage points; 95\% CI, 0.0 to 4.8) (Fig. S3 in the Supplementary Appendix). During follow-up, more participants in the placebo group (5.2\%) than in the vitamin D group (2.6\%) reported use of outside-of-trial vitamin D supplements above the 


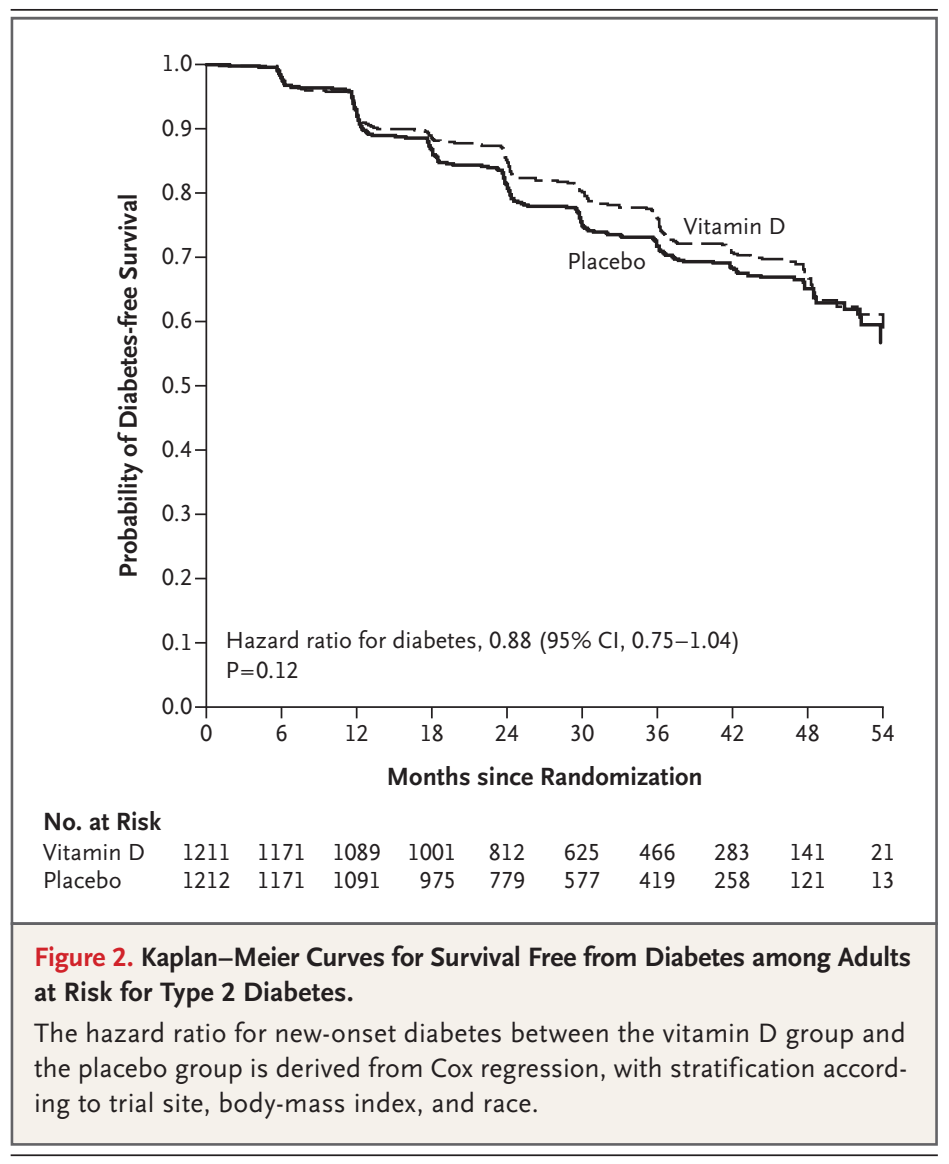

trial limit of 1000 IU per day (difference, 2.6 percentage points; 95\% CI, 1.1 to 4.2 ) (Fig. S2 in the Supplementary Appendix). There was no significant difference between the two groups in the use of outside-of-trial calcium supplements above the trial limit.

In the exploratory per-protocol analysis that censored follow-up data when a participant started a diabetes or weight-loss medication, stopped the trial pills, or took out-of-trial vitamin D from supplements above the trial limit of $1000 \mathrm{IU}$ per day, the primary outcome occurred in 265 participants $(21.9 \%)$ in the vitamin D group and $304(25.1 \%)$ in the placebo group (hazard ratio, 0.84 ; $95 \%$ CI, 0.71 to 1.00 ).

\section{SAFETY}

There were no significant between-group differences in the protocol-specified adverse events of interest: hypercalcemia, a fasting urine calcium:creatinine ratio of more than 0.375 , a low estimated glomerular filtration rate, and nephrolithiasis (Table 2). Overall, 47 participants (3.9\%) in the vitamin D group stopped the trial pills because of an adverse event, as compared with 37 (3.1\%) in the placebo group (difference, 0.8 percentage points; $95 \% \mathrm{CI},-0.7$ to 2.3 ).

\section{DISCUSSION}

In this multicenter, randomized, placebo-controlled trial involving persons at high risk for type 2 diabetes not selected for vitamin $\mathrm{D}$ insufficiency, vitamin $\mathrm{D}_{3}$ supplementation at a dose of 4000 IU per day did not result in a significantly lower risk of diabetes than placebo after a median follow-up of 2.5 years.

While our trial was being conducted, two other trials that were designed to test whether vitamin D supplementation lowers the risk of type 2 diabetes among persons at risk showed hazard ratios with vitamin $\mathrm{D}$ that were similar to those in our trial. ${ }^{25,26}$ In the Troms $\phi$ Vitamin D and T2DM Trial (Norway), which randomly assigned 511 white adults with prediabetes to 20,000 IU per week (approximately 2900 IU per day) of vitamin $\mathrm{D}_{3}$ or placebo, the risk of diabetes was numerically lower in the vitamin $\mathrm{D}$ group than in the placebo group, but the difference was not significant (hazard ratio, 0.90; 95\% CI, 0.69 to 1.18$).{ }^{25}$ In the Diabetes Prevention with Active Vitamin D study (Japan), which randomly assigned 1256 adults with prediabetes to an active form of vitamin D analogue (eldecalcitol) or placebo, the risk of diabetes was also lower in the vitamin $\mathrm{D}$ group than in the placebo group, but the difference was again not significant (hazard ratio, $0.87 ; 95 \%$ CI, 0.68 to 1.09 ). ${ }^{27}$ We powered our trial to detect a $25 \%$ lower risk of diabetes with vitamin $\mathrm{D}$ than with placebo. On the basis of the results from all three trials, vitamin D supplementation may decrease diabetes risk among persons at risk for diabetes not selected for vitamin D insufficiency by a smaller effect size (10 to $15 \%$ ), but none of these trials were powered to test this effect size.

Our trial has several strengths. We used contemporary glycemic criteria to assemble a diverse cohort at high risk for diabetes with a hyperglycemic pattern closely matching how prediabetes is diagnosed in clinical practice, most commonly with fasting plasma glucose and glycated hemoglobin. The vitamin D dose of 4000 IU per day 


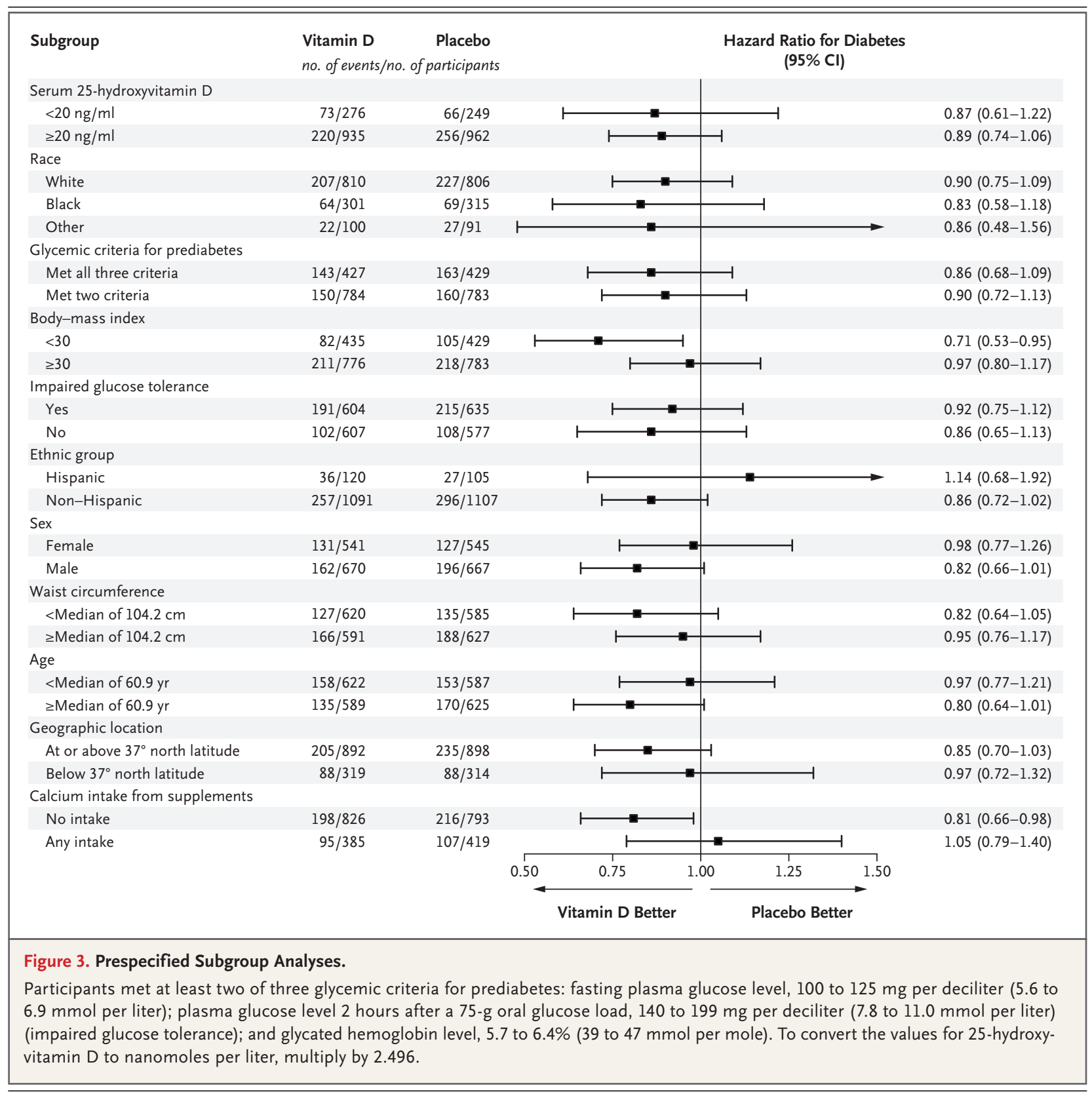

was selected to balance safety and efficacy and resulted in a large difference in the serum 25-hydroxyvitamin $\mathrm{D}$ level between the trial groups in the first 2 years of follow-up. In 94\% of cases, the primary outcome was ascertained by trial-specific laboratory testing based on current ADA criteria and required two tests in the diabetes range for diagnosis. Our cohort was recruited at a constant rate throughout the cal- endar year, which reduced the potential of confounding by seasonal variability. Finally, the observed rate of new-onset diabetes in the placebo group (10.7 events per 100 person-years) was consistent with our estimate of 10 events per 100 person-years.

Overall adherence was high, and overall use of off-protocol concomitant therapies was low. However, among nonadherent participants, more 


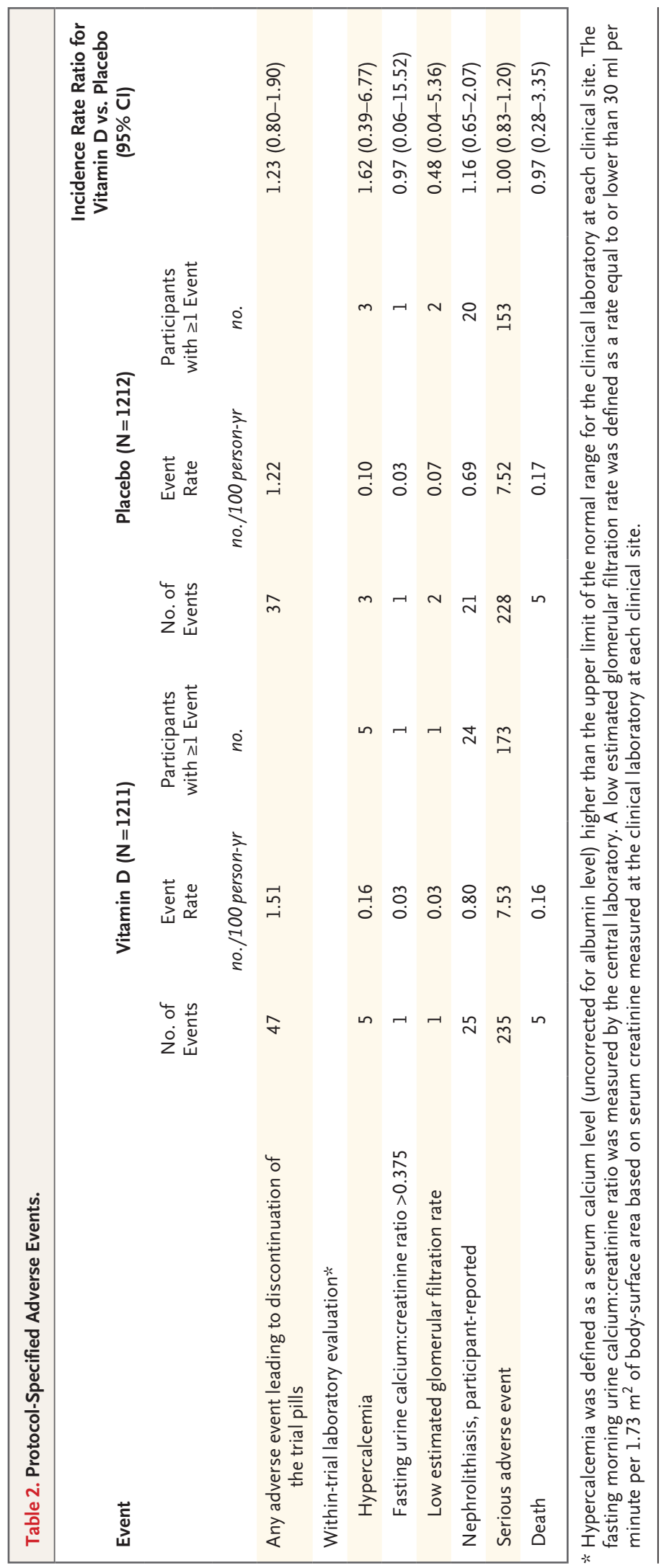

in the placebo group started diabetes or weightloss medications and took outside-of-trial vitamin D supplements above the trial limit, whereas more in the vitamin D group stopped the trial pills for any reason. Whether these differences among nonadherent participants shifted the risk difference between the two groups toward or away from null in the intention-to-treat or perprotocol analysis is unknown.

Response to a nutritional intervention depends on nutritional status at baseline; thus, if vitamin $\mathrm{D}$ has an effect on diabetes prevention, persons with a higher baseline level of serum 25-hydroxyvitamin D would be expected to have less effect from supplementation than those with a lower baseline level. ${ }^{28}$ Owing to ethical and practical considerations, a lack of consensus on the preferred 25-hydroxyvitamin D level, and our desire to maximize the external validity of the trial, we specifically did not include serum 25-hydroxyvitamin D as an eligibility criterion. Because vitamin D supplements are used increasingly in the U.S. adult population, ${ }^{29}$ approximately 8 of 10 participants had a baseline serum 25-hydroxyvitamin $\mathrm{D}$ level that was considered to be sufficient according to current recommendations ( $\geq 20 \mathrm{ng}$ per milliliter) to reduce the risk of many outcomes, ${ }^{23,30}$ including diabetes. ${ }^{6}$ The high percentage of participants with adequate levels of vitamin $D$ may have limited the ability of the trial to detect a significant effect.

The vitamin D dose of 4000 IU per day is the recommended upper intake level to avert potential toxicity, ${ }^{23}$ although data from large trials on the safety on this dose have been scant. There is concern that 25-hydroxyvitamin D levels above $50 \mathrm{ng}$ per milliliter (125 nmol per liter) may be associated with adverse effects. ${ }^{23,30}$ In our trial, vitamin $\mathrm{D}_{3}$ supplementation at a dose of $4000 \mathrm{IU}$ per day resulted in no significant differences between the two groups in the protocol-specified adverse events of interest (hypercalcemia, a fasting urine calcium:creatinine ratio of $>0.375$, a low estimated glomerular filtration rate, and nephrolithiasis). The 24-hour urine calcium level was not measured. ${ }^{31}$

In conclusion, among persons at high risk for type 2 diabetes not selected for vitamin $\mathrm{D}$ insufficiency, vitamin $\mathrm{D}_{3}$ supplementation at a dose of 4000 IU per day did not result in a significantly lower risk of diabetes than placebo. 
The views expressed in this article are those of the authors and do not necessarily represent the views of the National Institutes of Health.

A data sharing statement provided by the authors is available with the full text of this article at NEJM.org.

The planning phase of the D2d trial was funded by the National Institute of Diabetes and Digestive and Kidney Diseases (NIDDK) through a multicenter clinical study implementation planning grant to Tufts Medical Center in Boston (U34DK091958; principal investigator, Dr. Pittas). Planning was also supported, in part, by the Intramural Research Program of the NIDDK. The conduct of the trial was supported primarily by the NIDDK and the Office of Dietary Supplements of the National Institutes of Health through the multicenter clinical study cooperative agreement (U01DK098245; principal investigator, Dr. Pittas) to Tufts Medical Center, where the D2d Coordinating Center is based. The U01 grant mechanism establishes the NIDDK project scientist (Dr. Staten) as a member of the D2d Research Group. The trial also received secondary funding from the American Diabetes Association to Tufts Medical Center (1-14-D2d-01; principal investigator, Dr. Pittas). Educational materials were provided by the National Diabetes Education Program.

Dr. Dawson-Hughes reports receiving grant support, paid to Tufts University, from Pfizer and DSM and travel support from Abiogen Pharma; Dr. Aroda, receiving consulting fees, paid to her institution, from Adocia, grant support, paid to her institution, and consulting fees from AstraZeneca/Bristol-Myers Squibb, Novo Nordisk, and Sanofi, consulting fees from BD and Zafgen, and grant support, paid to her institution, from Calibra Medical, Eisai, Janssen, and Theracos; Dr. Ceglia, receiving grant support from DSM; Dr. Desouza, receiving advisory board fees from Novo Nordisk; Dr. Kim, receiving consulting fees from Sanofi; Dr. LeBlanc, receiving grant support, paid to her institution, from Merck; Dr. Neff, receiving grant support from GI Dynamics; Dr. O'Neil, receiving grant support from Weight Watchers International, advisory board fees from Janssen, advisory board fees and fees for presentations from Vindico Medical Education, fees for CME programs from WebMD, lecture fees from Robard, and grant support and lecture fees from Novo Nordisk; Dr. Phillips, receiving grant support and advisory board fees from Janssen Pharmaceuticals, receiving advisory board fees from Profil Institute for Clinical Research, receiving grant support from Merck, Amylin Pharmaceuticals, Eli Lilly, Novo Nordisk, Sanofi, PhaseBio, Roche, AbbVie, Vascular Pharmaceuticals, GlaxoSmithKline, Pfizer, and Kowa Research Institute, and serving as cofounder, officer, and board member of and holding stock in Diasyst; and Dr. Pratley, receiving lecture fees, paid to his institution, and consulting fees, paid to his institution, from AstraZeneca, consulting fees, paid to his institution, from Boehringer Ingelheim, Eisai, GlaxoSmithKline, Glytec, Janssen, Mundipharma, and Pfizer, grant support, paid to his institution, from Lexicon Pharmaceuticals, grant support, paid to his institution, and consulting fees, paid to his institution, from Ligand Pharmaceuticals, Eli Lilly, Merck, and Sanofi, grant support, paid to his institution, and lecture fees, paid to his institution, from Novo Nordisk and Takeda, and consulting fees from Sanofi US Services. No other potential conflict of interest relevant to this article was reported.

Disclosure forms provided by the authors are available with the full text of this article at NEJM.org.

We thank the D2d investigators, staff, and trial participants for their dedication and commitment to the trial.

\section{APPENDIX}

The authors' affiliations are as follows: Tufts Medical Center (A.G.P., L.C., P.F., J.N., E.M.V.), the Jean Mayer USDA Human Nutrition Research Center on Aging at Tufts University (B.D.-H.), Brigham and Women's Hospital (V.R.A.), and Harvard School of Public Health (J.H.W.), Boston, and the Spaulding Rehabilitation Network, Charlestown (P.S.) — all in Massachusetts; National Institute of Diabetes and Digestive and Kidney Diseases, Phoenix, AZ (W.C.K.); the Maine Medical Center (I.B.) and the Maine Medical Center Research Institute (C.R.) — both in Scarborough; HealthPartners Institute, Minneapolis (C.C.); Duke University Medical Center, Durham, NC (R.C., R.D.); the University of Nebraska Medical Center and Omaha Veterans Affairs Medical Center, Omaha (C.D.); Baylor College of Medicine, Houston (J.F.), and the University of Texas Southwestern Medical Center, Dallas (P.R.) - both in Texas; MedStar Good Samaritan Hospital, Baltimore (A.G.), MedStar Health Research Institute, Hyattsville (J.P.), and the National Institute of Diabetes and Digestive and Kidney Diseases, Bethesda (M.S.) — all in Maryland; Pennington Biomedical Research Center, Baton Rouge, LA (D.S.H.); the University of Tennessee Health Science Center, Memphis (K.C.J.); Cleveland Clinic, Cleveland (S.R.K.); Stanford University Medical Center, Stanford (S.K.), and the Keck School of Medicine of the University of Southern California, Los Angeles (A.P.) — both in California; Kaiser Permanente Center for Health Research-Northwest, Portland, OR (E.S.L.); the University of Vermont, Burlington (M.R.L.); Northwell Health Lenox Hill Hospital, New York (E.L.); Northwestern University, Chicago (L.M.N.); the Medical University of South Carolina, Charleston (P.O.); Emory University School of Medicine, Atlanta, and the Atlanta Veterans Affairs Medical Center, Decatur — both in Georgia (L.S.P.); AdventHealth Translational Research Institute for Metabolism and Diabetes, Orlando, FL (R.P.); the University of Colorado Denver and the Veterans Affairs Eastern Colorado Health Care System, Denver (N.R.); and the University of Kansas Medical Center, Kansas City (D.R.).

\section{REFERENCES}

1. Centers for Disease Control and Prevention. National diabetes statistics report, 2017 (https://www.cdc.gov/diabetes/pdfs data/statistics/national-diabetes-statistics -report.pdf).

2. Knowler WC, Barrett-Connor E, Fowler $\mathrm{SE}$, et al. Reduction in the incidence of type 2 diabetes with lifestyle intervention or metformin. N Engl J Med 2002;346: 393-403.

3. Lu L, Bennett DA, Millwood IY, et al. Association of vitamin $\mathrm{D}$ with risk of type 2 diabetes: a Mendelian randomisation study in European and Chinese adults. PLoS Med 2018;15(5):e1002566.
4. Pittas AG, Lau J, Hu FB, DawsonHughes B. The role of vitamin D and calcium in type 2 diabetes: a systematic review and meta-analysis. J Clin Endocrinol Metab 2007;92:2017-29.

5. Kayaniyil S, Vieth R, Retnakaran R, et al. Association of vitamin D with insulin resistance and beta-cell dysfunction in subjects at risk for type 2 diabetes. Diabetes Care 2010;33:1379-81.

6. Song Y, Wang L, Pittas AG, et al. Blood 25-hydroxy vitamin D levels and incident type 2 diabetes: a meta-analysis of prospective studies. Diabetes Care 2013; 36:1422-8
7. Mitri J, Dawson-Hughes B, Hu FB, Pittas AG. Effects of vitamin D and calcium supplementation on pancreatic $\beta$ cell function, insulin sensitivity, and glycemia in adults at high risk of diabetes: the Calcium and Vitamin D for Diabetes Mellitus (CaDDM) randomized controlled trial. Am J Clin Nutr 2011;94:486-94.

8. Seida JC, Mitri J, Colmers IN, et al. Effect of vitamin D3 supplementation on improving glucose homeostasis and preventing diabetes: a systematic review and meta-analysis. J Clin Endocrinol Metab 2014;99:3551-60.

9. Mirhosseini N, Vatanparast $\mathrm{H}$, Mazidi 
M, Kimball SM. Vitamin D supplementation, glycemic control, and insulin resistance in prediabetics: a meta-analysis. J Endocr Soc 2018;2:687-709.

10. Tang H, Li D, Li Y, Zhang X, Song Y, Li X. Effects of vitamin D supplementation on glucose and insulin homeostasis and incident diabetes among nondiabetic adults: a meta-analysis of randomized controlled trials. Int J Endocrinol 2018;2018: 7908764.

11. Pittas AG, Dawson-Hughes B, Sheehan $\mathrm{PR}$, et al. Rationale and design of the Vitamin D and Type 2 Diabetes (D2d) study: a diabetes prevention trial. Diabetes Care 2014;37:3227-34.

12. American Diabetes Association. Standards of medical care in diabetes 2010. Diabetes Care 2010;33:Suppl 1:S11S61.

13. Aroda VR, Sheehan PR, Vickery EM, et al. Establishing an electronic health record-supported approach for outreach to and recruitment of persons at high risk of type 2 diabetes in clinical trials: the Vitamin D and Type 2 diabetes (D2d) study experience. Clin Trials 2019;16: 308-15.

14. Gökçe C, Gökçe O, Baydinç C, et al. Use of random urine samples to estimate total urinary calcium and phosphate excretion. Arch Intern Med 1991;151:1587-8. 15. Matsushita K, van der Velde M, Astor $\mathrm{BC}$, et al. Association of estimated glomerular filtration rate and albuminuria with all-cause and cardiovascular mortal- ity in general population cohorts: a collaborative meta-analysis. Lancet 2010;375: 2073-81.

16. Forrest RD, Jackson CA, Yudkin JS. The glycohaemoglobin assay as a screening test for diabetes mellitus: the Islington Diabetes Survey. Diabet Med 1987;4:254-9. 17. Bedner M, Lippa KA, Tai SS. An assessment of 25-hydroxyvitamin D measurements in comparability studies conducted by the Vitamin D Metabolites Quality Assurance Program. Clin Chim Acta 2013; 426:6-11.

18. DEQAS (Vitamin D External Quality Assessment Scheme) (http://www.deqas.org). 19. Schoenfeld DA. Sample-size formula for the proportional-hazards regression model. Biometrics 1983;39:499-503.

20. Haybittle JL. Repeated assessment of results in clinical trials of cancer treatment. Br J Radiol 1971;44:793-7.

21. Lachin JM. Maximum information designs. Clin Trials 2005;2:453-64.

22. Cox DR. Regression models and life tables. J R Stat Soc [B] 1972;34:187-220.

23. Institute of Medicine. Dietary reference intakes for calcium and vitamin D. Washington, DC: National Academies Press, 2011.

24. LeBlanc ES, Pratley RE, DawsonHughes B, et al. Baseline characteristics of the Vitamin D and Type 2 Diabetes (D2d) study: a contemporary prediabetes cohort that will inform diabetes prevention efforts. Diabetes Care 2018;41:1590-9. 25. Jorde R, Sollid ST, Svartberg J, et al.
Vitamin D 20,000 IU per week for five years does not prevent progression from prediabetes to diabetes. J Clin Endocrinol Metab 2016;101:1647-55.

26. Kawahara T, Suzuki G, Inazu T, et al. Rationale and design of Diabetes Prevention with active Vitamin D (DPVD): a randomised, double-blind, placebo-controlled study. BMJ Open 2016;6(7):e011183.

27. Kawahara T. Eldecalcitol, a vitamin D analog, for diabetes prevention in impaired glucose tolerance (DPVD study). Diabetes 2018;67:Suppl 1 (poster) (http:/l diabetes.diabetesjournals.org/content/67/ Supplement_1/120-LB).

28. Grant WB, Boucher BJ, Bhattoa HP, Lahore $\mathrm{H}$. Why vitamin $\mathrm{D}$ clinical trials should be based on 25-hydroxyvitamin D concentrations. J Steroid Biochem Mol Biol 2018;177:266-9.

29. Rooney MR, Harnack L, Michos ED, Ogilvie RP, Sempos CT, Lutsey PL. Trends in use of high-dose vitamin D supplements exceeding 1000 or 4000 international units daily, 1999-2014. JAMA 2017; 317:2448-50.

30. National Institutes of Health. Vitamin D: fact sheet for health professionals (https:/l ods.od.nih.gov/factsheets/VitaminD -HealthProfessional/).

31. Jones AN, Shafer MM, Keuler NS, Crone EM, Hansen KE. Fasting and postprandial spot urine calcium-to-creatinine ratios do not detect hypercalciuria. Osteoporos Int 2012;23:553-62.

Copyright (C) 2019 Massachusetts Medical Society. 